\title{
POUVOIR DISCIPLINAIRE ET NORMALISATION
}

Le début du XIXe siècle marque, selon Michel Foucault, une période de mutations fondamentales dans la redistribution de l'économie des châtiments. La disparition du "châtiment-spectacle"1 représente l'un des événements les plus importants de ce processus de transformation. L'art de punir, qui se caractérise par sa discrétion, se charge, selon les réformistes, de plus en plus d'humanité. La perspective que Foucault adopte remet en cause, de manière implicite et à travers une description du redéploiement des stratégies punitives, les thèses réformistes. L'objectif de Foucault, qui sera aussi celui de la présente étude, est d'entreprendre une "histoire corrélative de l'âme moderne et d'un nouveau pouvoir de juger, une généalogie de l'actuel complexe scientifico-judiciaire où le pouvoir de punir prend ses appuis, reçoit ses justifications et ses règles, étend ses effets et masque son exhorbitante singularité" (27). La définition de la société disciplinaire, l'inventaire des pratiques non-discursives (telles l'architecture) et discursives qui composent le pouvoir disciplinaire, l'anlayse des moyens du bon redressement et plus particulièrement de la norme et de la normalisation constituent les différents points qui seront abordés.

La société disciplinaire s'élabore vers la fin du XVIIle et au début du XIXe siècle. Elle se caractérise par un art de punir qui traverse le corps de manière spécifique et nouvelle basée sur le contrôle et la surveillance. Le nouvel art de punir qui s'élabore dans le régime du pouvoir disciplinaire ne vise pas seulement la répression. Foucault nous apprend que la punition disciplinaire met en oeuvre une série de cinq opérations bien distinctes. La première consiste à référer les actes, les performances et les conduites singulières à "un ensemble qui est à la fois champ de comparaison, espace de différentiation et principe d'une règle à suivre". Cette règle ou norme va ensuite servir à différencier les individus les uns par rapport aux autres. Les troisième et quatrième principes consistent à mesurer en termes quantitatifs et de hiérarchiser "en termes de valeurs les capacités, le niveau, la "nature" des individus; à "faire jouer, à travers cette mesure "valorisante", la contrainte d'une conformité à réaliser" (182). La dernière règle définit la frontière extérieure de

${ }^{1}$ Foucault, Surveiller et punir, p. 15. Les citations qui suivront seront indiquées entre parenthèses. 
l'anormal, permettant ainsi au châtiment disciplinaire de réduire les écarts par rapport à la norme. La société disciplinaire fonctionne sur la base d'un pouvoir essentiellement correctif. Foucault situe la société disciplinaire au moment de la formation des disciplines. Il écrit notamment:

\begin{abstract}
Le moment historique des disciplines, c'est là où naît un art du corps humain, qui ne vise pas seulement à la croissance de ses habilétés, ni non plus l'alourdissement de sa sujétion, mais la formation d'un rapport qui dans le mécanisme le rend d'autant plus obéissant qu'il est plus utile, et inversement. Se forme alors une politique des coercitions qui sont un travail sur le corps, une manipulation calculée de ses éléments, de ses gestes, de ses comportements. Le corps humain entre dans une machinerie du pouvoir qui le fouille, le désarticule et le recompose.
\end{abstract}

La société disciplinaire secrète une "machinerie de contrôle" qui fonctionne comme un microscope de la conduite. Le corps humain est ainsi sous l'emprise d'une observation permanente et méticuleuse, microscopique. L'appareil d'observation par excellence va être le regard. Toute une problématique se développe alors:

celle d'une architecture qui n'est plus faite seulement pour être vue (fastes du palais), ou pour surveiller l'espace extérieur (géométries des forteresses), mais pour permettre un contróle intérieur, articulé et détaille--pour rendre visibles ceux qui s'y trouvent; plus généralement, celle d'une architecture qui serait un operateur pour la transformation des individus: agir sur ceux qu'elle abrite, donner prise sur leur conduite, reconduire jusqu'à eux les effets de pouvoir, les offrir à une connaissance et les modifier.

La répartition des individus dans l'espace disciplinaire se fait selon des techniques telles que la clôture qui emprunte sa forme au couvent, l'internat qui devient la norme dans l'éducation, les casernes militaires qui enferment les militaires tout en les plaçant, de jour comme de nuit, sous le regard des officiers chargés du contrôle. La surveillance hiérarchique ne s'opère pas seulement dans l'espace. L'emprise du regard va aussi s'exercer au niveau de l'âme qui est soumise à un savoir hiérarchisant. Le regard du surveillant contraint les élèves et tous ceux qui sont vus à le considérer comme référence ultime dans l'ordre du savoir. En se constituant comme norme de savoir, le regard disciplinaire légitime sa position hiérarchique. Comme l'écrit Foucault: 
L'appareil disciplinaire parfait permettrait à un seul regard de tout voir en permanence. Un point central serait à la fois source de lumière éclairant toutes choses, et lieu de convergence pour tout ce qui doit être su: oeil parfait auquel rien n'échappe et centre vers lequel tous les regards sont toumés.

Dans le système scolaire, 1' "école-bâtiment doit être un opérateur de dressage" (175). Foucault constate que l'espace de la classe va devenir homogène, permettant ainsi aux élèves d'être placés sous le regard du maître. Le principe de clôture est doublé par celui du quadrillage qui consiste à localiser chaque individu en lui attribuant une place précise: "L'espace disciplinaire, écrit Foucault, tend à se diviser en autant de parcelles qu'il y a de corps ou d'éléments à répartir" (144). La recomposition de l'architecture par le pouvoir disciplinaire a pour fonction de produire des corps et des sujets dociles. L'architecture, comme le montre Foucault, devient une composante essentielle de la société de contrôle. Les mutations architecturales font partie des pratiques non-discursives auxquelles a recours la société disciplinaire pour imposer sa logique de pouvoir. L'analyse va maintenant explorer les pratiques discursives du pouvoir disciplinaire. Le discours de normalité qui s'élabore dans ce que Foucault appelle le complexe scientifico-juridique, le processus de normalisation constituent les différentes articulations des prochaines lignes. Mais il convient tout d'abord de définir la norme et les rapports qu'elle entretient avec les sciences humaines.

Le normal est traditionnellement perçu comme une loi de la nature. Il est aussi perçu comme quelque chose qui découle des lumières de la raison, des critères logiques et qui a une base rationnelle. La norme se présente toujours comme une sorte d'évidence reconnue par tous. Le normal fonctionne comme une référence qui va de soi. Foucault, dans la description du processus d'élaboration des normes, va implicitement déconstruire cette perception traditionnelle. La première stratégie à laquelle Foucault a recours pour renverser la perception commune de la notion de norme est le dévoilement de son mécanisme correctif. Foucault estime que la norme est un instrument du pouvoir disciplinaire. Selon Foucault, la norme vient s'ajouter à d'autres pouvoirs tels celui de la Loi, celui de la Parole et du Texte, celui de la Tradition. Foucault définit le pouvoir de la norme comme la nouvelle loi de la société moderne. Il écrit:

Le Normal s'établit comme principe de coercition dans l'enseignement avec l'instauration d'une éducation standardisée et l'établissement des écoles normales; il s'établit dans l'effort pour organiser un corps médical et un encadrement hospitalier de la nation susceptibles de faire fonctionner des normes générales; il s'établit dans la 
régularisation des produits industriels. Comme la surveillance et avec elle, la normalisation devient un des grands instruments de pouyoir à la fin de l'âge classique.

(186)

La deuxième stratégie utilisée par Foucault consiste à remettre en cause le caractère naturel de la norme. Foucault démontre que la norme n'a aucune matérialité, aucune légitimité naturelle. Au contraire, la norme est une construction qui se forme dans le "pouvoir d'écriture" (191). La norme est une formalisation des pratiques discursives et non discursives de la société disciplinaire. A propos du processus qui mène à l'établissement des normes dans le domaine scolaire, Foucault ajoute:

Les autres innovations de l'ecriture disciplinaire concernent la mise en corrélation de ces éléments, le cumul des documents, leur mise en série, l'organisation de champs comparatifs permettant de classer, de former des catégories, d'établir des moyennes, de fixer des norm(d囚2)

La norme émerge donc de manière tout à fait contingente d'une pratique qui se développe sans orientation théorique préalable. La norme surgit de l'examen documentaire. Elle n'est pas antérieure aux pratiques documentaires du sytème scolaire. La norme ne saurait donc être un avant, elle est toujours un après que la société disciplinaire naturalise ou rationalise après coup.

L'élaboration de la norme a, selon Foucault, une importance décisive dans l'ouverture épistémologique de l'espace qui sera celui des sciences de l'individu. L'apparition de la norme va permettre la constitution des sciences humaines. La norme fonctionne ainsi comme un mécanisme de subjectivation, comme un moyen de produire des sujets contrôlables par le régime disciplinaire. Il est significatif de constater que la norme et la notion d'humanisme émergent presque au même moment. L'humanisme est une tentative d'imposer un modèle d'humanité universelle.

La formation de la norme constitue le moment historique de l'entrée de l'individu dans le champ du savoir. A la question aristotélicienne de savoir si une science de l'individu serait possible et légitime, Foucault formule la réponse suivante:

Il faut regarder du côté de ces procédures d'écriture et d'enregistrement, il faut regarder du côté des mécanismes d'examen, du côté de la formation des dispositifs de discipline, et de la formation d'un nouveau type de pouvoir sur les corps. La naissance des sciences de l'homme? Elle est vraisemblablement à chercher dans ces archives de peu de gloire où s'est élaboré le jeu moderne des coercitions sur les 
L'entrée de "l'homme" dans le fonctionnement général du discours scientifique le constitue en objet de savoir et instaure ce que Foucault appelle une nouvelle technologie du pouvoir: 1 ' "examen est au centre des procédures qui constituent l'individu comme effet et objet de pouvoir, comme effet et objet de savoir" (174). Le pouvoir disciplinaire, à travers l'examen qui combine la surveillance hiérarchique et la sanction normalisatrice, fait de chaque individu un "cas" qui constitue à la fois un objet de connaissance et une prise du pouvoir. Pour Foucault, le cas n'est plus seulement, comme dans la casuistique ou la jurisprudence, un ensemble de circonstances "qualifiant un acte et pouvant modifier l'application d'une règle, c'est l'individu tel qu'on peut le décrire, le jauger, le mesurer, le comparer à d'autres et cela dans son individualité même; et c'est aussi l'individu qu'on a à dresser ou redresser, qu'on a à classer, à normaliser, à exclure" (193). La formation de la norme permet donc en même temps de poser les modèles du conformisme, de définir les individus différenciés. Elle institue le processus d'uniformisation, c'est-à-dire la normalisation.

$\mathrm{Au}$ cours des réformes qui structurent les bases du pouvoir disciplinaire, Foucault remarque qu'une armée de techniciens vient prendre la rélève du bourreau. Il s'agit des surveillants, des médecins, des aumôniers, des psychologues et des psychiatres. Le psychiatre, qui nous intéresse particulièrement à cause du concept de folie, remplace ainsi le bourreau qui infligeait les supplices dans la société répressive. Le psychiatre représente dès lors l' "agent de la non-souffrance" (17) dans les mécanismes de punition de la société disciplinaire. Avec la psychiatrie, la punition va se déplacer progressivement vers l'âme. Foucault écrit à ce propos:

\footnotetext{
Dans tout le rituel pénal, depuis l'information jusqu'à la sentence et les dernières séquelles de la peine, on a fait pénétrer un domaine d'objets qui viennent doubler, mais aussi dissocier les objets juridiquement définis et codés. L'expertise psychiatrique, mais d'une façon plus générale l'anthropologie criminelle et le rassassant discours de la criminologie trouvent là une de leurs fonctions précises.

La psychiatrie va devenir un des instruments du pouvoir disciplinaire en cours de formation. Elle vient cependant se juxtaposer à la pénalité judiciaire; ce qui ne va pas sans créer des ambiguités. La juxtaposition du pouvoir disciplinaire (pouvoir correctif) et de la pénalité judiciaire (pouvoir répressif) provoque des modifications dans le fonctionnement de la justice. 
Le jugement ne détermine plus simplement la vérité d'un crime à partir de la connaissance de l'infraction, de l'accusé et de la loi. Le jugement devient appréciatif, diagnostiqueur et normatif. La dimension normative du jugement se manifeste dans l'évolution du traitement de la folie dans la pratique pénale. Foucault écrit:

\begin{abstract}
D'après le Code de 1810, elle n'était posée qu'au terme de l'article 64. Or celui-ci porte qu'il n'y a ni crime ni délit, si l'infracteur était en état de démence au moment de l'acte. La possibilité d'assigner la folie était done exclusive de la qualification d'un acte comme crime: que l'auteur ait été fou, ce n'était pas la gravité de son geste qui était modifiée, ni sa peine qui devait en étre atténuée; le crime lui-même disparaissait. Impossible donc de déclarer quelqu'un coupable et fou; le diagnostic de folie s'il était posé ne pouvait pas s'intégrer au jugement; il interrompait la procédure, et dénouait la prise de justice sur l'auteur de l'acte.
\end{abstract}

(24-25)

On remarque cependant un décalage entre la loi et les tribunaux. En effet, la question de la folie est en permanence au coeur des verdicts: "Ils ont admis qu'on pouvait être coupable et fou; d'autant moins coupable qu'on était un peu plus fou; coupable certes, mais à enfermer plutôt qu'à punir" (25). Ainsi une expertise psychiatrique concluante soustrait l'accusé au pouvoir disciplinaire pour le soumettre aux effets du pouvoir disciplinaire. Ce qui est nouveau ici, c'est que l'expertise psychiatrique et le diagnostic de folie sont des expressions qui rélèvent d'un discours de vérité, d'une connaissance scientifique de la folie. La métamorphose des méthodes punitives institue ici de nouvelles relations entre le savoir et le pouvoir. Pour Foucault, l'homme, l'âme, l'individunormal ou anormal en venant doubler le crime comme objets de l'intervention pénale, sont pris dans un mode spécifique d'assujétissement qui "a pu donner naissance à l'homme comme objet de savoir pour un discours à statut "scientifique" (29). D'après, Foucault l'intrusion du principe de normalité dans l'univers judiciaire provoque un morcellement du pouvoir légal de punir entre une série d'instances annexes. Le psychiatre impose une orientation toute nouvelle à la justice criminelle. Foucault voit en lui un juge annexe, un "conseiller en punition":

Quant aux experts psychiatriques, ils peuvent bien se défendre de juger. Qu'on examine les trois questions auxquelles, depuis la circulaire de 1958, ils ont à répondre: l'inculpé présente-t-il un état de danger? Est-il curable à la sanction pénale? Est-il curable ou réadaptable? Ces questions n'ont pas de rapport avec l'article 64, ni avec la folie éventuelle de l'inculpé au moment de l'acte. Ce sont des 
questions en termes de "responsabilite". Elles ne concement que l'administration de la peine, sa nécessité, son utilité, son efficace possible; elles permettent d'indiquer, dans un vocabulaire à peine codé, si l'asile vaut mieux que la prison.

Il convient toutefois de relever le phénomène suivant: l'expertise psychiatrique interrompt ou alors influence la procédure judiciaire. Le processus thérapeutique découle quant à lui de la sanction pénale. Mais le "fou" va être condamné à être soigné ou normalisé, processus qui fait partie du pouvoir disciplinaire. La pratique de l'expertise psychiatrique fait que la sentence, "même si elle est toujours formulée en termes de sanction légale, implique, plus ou moins obscurément des jugements de normalitén (25). La sentence pénale se mêle ainsi d'éléments non juridiques qui vont du diagnostic des anomalies à la normalisation:

Au lieu que la folie efface le crime au sens premier de l'article 64 , tout crime maintenant et, à la limite, toute infraction portent en soi, comme un droit qu'ils peuvent revendiquer, l'hypothèse de la folie, en tout cas de l'anomalie. Et la sentence qui condamne ou acquitte n'est pas seulement un jugement de culpabilité, une décision légale qui sanctionne; elle porte avec elle une appreciation de normalité et une prescription technique pour une normalisation possible.

La normalisation peut se définir comme l'obligation de se conformer à des normes de conduite. Foucault la définit comme un "processus de dressage et de correction" (182). La normalisation impose à des individus préalablement repertọiés comme anormaux une "procédure d'objectivation et d'assujetissement" (193) destinée à produire des sujets normaux. La normalisation peut donc être considérée comme une entreprise de subjectivation, "processus par lequel on obtient la constitution d'un sujet, plus exactement d'une subjectivité". ${ }^{2}$ La normalisation suppose deux opérations contradictoires. Elle vise l'homogénéisation des conduites. Mais en même temps, elle établit une différentiation des individus afin de mieux les contrôler, de mieux les assujetir à la norme:

En un sens le pouvoir de normalisation contraint à l'homogéneité, mais il individualise en permettant de mesurer les écarts, de déterminer les niveaux, de fixer les spécialités et de rendre les différences utiles en les ajustant les unes aux autres.

La normalisation s'inscrit dans le mouvement plus global de la

${ }^{2}$ Foucault, Interview par Gilles Barbedette et André Scala, p. 41. 
généralisation de la punition. Elle fonctionne comme une annexe de la pénalité judiciaire tout en investissant les domaines qui ne sont pas pris en charge par la loi. Le domaine couvert par la normalisation est ainsi illimité: "Est pénalisable le domaine indéfini du non-conforme". La normalisation reconduit ce que Foucault appelle un "rituel de pouvoir" (188). Foucault pense qu'il existe des connections entre la normalisation qui surgit au XIXe siècle et les mécanismes de pouvoir utilisés au Moyen-âge contre les lépreux:

Le partage constant du normal et de l'anormal, auquel tout individu est soumis, reconduit jusqu'à nous et en les appliquant à de tout autres objets, le marquage binaire et l'exil du lépreux; l'existence de tout un ensemble de techniques et d'institutions qui se donnent pour táche de mesurer, de contrôler, et de corriger les anormaux, fait fonctionner les dispositifs disciplinaires qu'appelait la peur de la peste. Tous les mécanismes de pouvoir qui, de nos jours encore, se disposent autour de l'anormal, pour le marquer comme pour le modifier, composent ces deux formes dont elles dérivent de loin.

La sanction normalisatrice est le point de départ d'un nouveau pouvoir de punir que Foucault qualifie de "juridicoanthropologique" (186). La pénalité moderne se forme ainsi à partir d'une technique disciplinaire dont la norme est l'une des composantes fondamentales.

En définitive, le pouvoir disciplinaire n'est pas seulement représsif, mais aussi productif. La norme ne reproduit pas le pouvoir judiciaire, elle fonctionne comme annexe de la loi et va finir par investir les appareils extérieurs. La normalisation constitue un processus de création des sujets conformes à une norme préalablement établie par les mécanismes de pouvoir. La normalisation travaille à un processus d'intériorisation des normes conventionnelles par les individus, permettant ainsi au pouvoir d'avoir une prise plus étroite sur le corps à travers l'âme. Le pouvoir disciplinaire "produit du réel; il produit des domaines d'objets et des rituels de vérité. L'individuet la connaissance qu'on peut en prendre rélèvent de cette production" (196): il institue ainsi de nouvelles relations entre le savoir et le pouvoir. La production du savoir venant légitimer les effets du pouvoir. 


\section{Textes consultés}

Foucault, Michel. Histoire de la folie d l'age classique. Paris: Gallimard,

1972.

-.-. Surveiller et punir. Naissance de la prison. Paris: Gallimard, 1975.

---. Interview par Gilles Barbedette et André Scala, Les Nouvelles, 28 juin au 5 juillet 1984, 36-41.

Shumway, David R. Michel Foucault. Charlotteville and London: UP of Virginia.

The Ohio State University

CILAS KEMEDJO 